\title{
Nonlinear optics in semiconductor optical fibers
}

\author{
Anna C. Peacock \\ Optoelectronics Research Centre, University of Southampton \\ Southampton SO17 1BJ, United Kingdom \\ acp@orc.soton.ac.uk
}

\begin{abstract}
This paper will review progress in the development of nonlinear devices from the semiconductor optical fibre platform. The nonlinear performance will be benchmarked through demonstrations of high-speed all-optical wavelength conversion, modulation, and continuum generation across a broad wavelength range.

Index Terms-Nonlinear Fiber Optics; Fiber Fabrication; Silicon Photonics
\end{abstract}

\section{INTRODUCTION}

The nascent field of semiconductor core fibers is attracting increased interest as a means to exploit the optoelectronic functionality of the semiconductor materials directly within the fiber geometry [1], [2]. Compared to their planar counterparts, this new class of waveguide retains many of the advantageous properties of the robust and flexible fiber platforms. Moreover, by making use of the two dimensional microstructured fiber templates, or by employing standard fiber post-processing procedures such as tapering, it is also possible to tailor the waveguide design far beyond what is achievable on-chip, of particular use for nonlinear applications [3].

In this paper, I will review our efforts regarding the optical characterization of a range of semiconductor fibers. Results of transmission measurements obtained for fibers with different core materials and geometries will be presented, with the potential to extend their application into the long (mid to far-infrared) wavelength regimes being discussed. Our work in this area has shown that this new fiber technology has great potential for the development of a wide range of devices including broadband sources, modulators, and amplifiers.

\section{FIBER FABRICATION}

There are two main approaches to fabricating semiconductor optical fibers. The first makes use of a high pressure chemical vapour deposition (HPCVD) method in which the semiconductor material is deposited inside micrometer sized pores of pre-fabricated capillaries [2]. This deposition-based method is quite flexible as it can be adapted to fill a range of silica templates with both amorphous and polycrystalline materials simply by tuning the deposition temperature and pressure. The second approach is based on a conventional fiber drawing method whereby the semiconductor material is packed inside a glass tube to create a millimeter sized preform, which is then heated and drawn down into a fiber with micrometer dimensions [1]. Owing to the high temperatures used in the drawing process, this method is currently restricted to producing polycrystalline fibers with core diameters on the order of ten micrometers or larger. Thus, in order to access the full range of materials and dimensions, our work makes use of both fiber types.

Fig. 1 displays microscope images of semiconductor fibers fabricated via the different techniques. Fig. 1(a) shows two fibers, an amorphous silicon microstructured fiber and a simple step index core fiber, fabricated via the HPCVD method. Fig. 1(b) shows a polysilicon fiber fabricated via the MCD approach that has been tapered, post-fabrication, to vary the core size along the length. The inset in Fig. 1(b) shows the etched core of a HPCVD ZnSe fiber for use as a cylindrical whispering gallery mode (WGM) resonator. For reference, typical loss values for these core materials are: $\sim 1 \mathrm{~dB} / \mathrm{cm}$ for hydrogenated amorphous silicon (a-Si:H), $\sim 1.5 \mathrm{~dB} / \mathrm{cm}$ for polysilicon (poly-Si), and $\sim 1 \mathrm{~dB} / \mathrm{cm}$ for $\mathrm{ZnSe}$ at $1.5 \mu \mathrm{m}$ [2], [4].
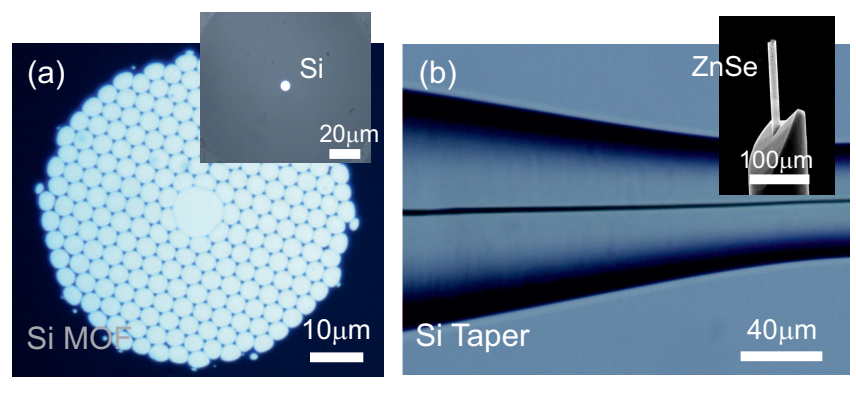

Fig. 1. Semiconductor core fibers. (a) Silicon microstructured optical fiber; inset shows a step index silicon core fiber. (b) Tapered silicon core fiber; inset shows a fiber-based $\mathrm{ZnSe}$ microcylindrical resonator.

\section{RESUltS AND DiscUSSION}

\section{A. Silicon Fibers}

Although these loss values are still high for an optical fiber, owing to the large nonlinear coefficients of the semiconductor materials they are nevertheless sufficient to allow for the first demonstrations of nonlinear propagation and characterization of this fiber class. To illustrate this, Fig. 2 shows examples of supercontinuum generation obtained in both the HPCVD a-Si:H and MCD poly-Si core materials. Fig. 2(a) shows two broadband spectra generated via a cascaded four-wave mixing process in the a-Si:H core fiber. A key advantage of this material is that, compared to poly-Si, it has a higher nonlinear coefficient and lower loss. For the longer pump wavelength we have obtained a continuum spanning more than an octave, which we attribute to the higher nonlinear figure of merit of 
the material as we move deeper into the mid-infrared [5]. Fig. 2(a) shows spectra generated in the poly-Si core fiber, which we believe to be the first observation of supercontinuum in this material system [6]. Although the nonlinearity in this fiber is lower than in (a), the length can be made much longer to compensate for this, in this case $\sim 5$ times longer. To further enhance the nonlinear process, the fiber was tapered to both reduce the core size and improve the crystallinity [7]. Owing to the longer fiber length, in this case the broadening is produced via soliton fission and again an octave-spanning continuum has been achieved for the longest pump wavelength.

(a)
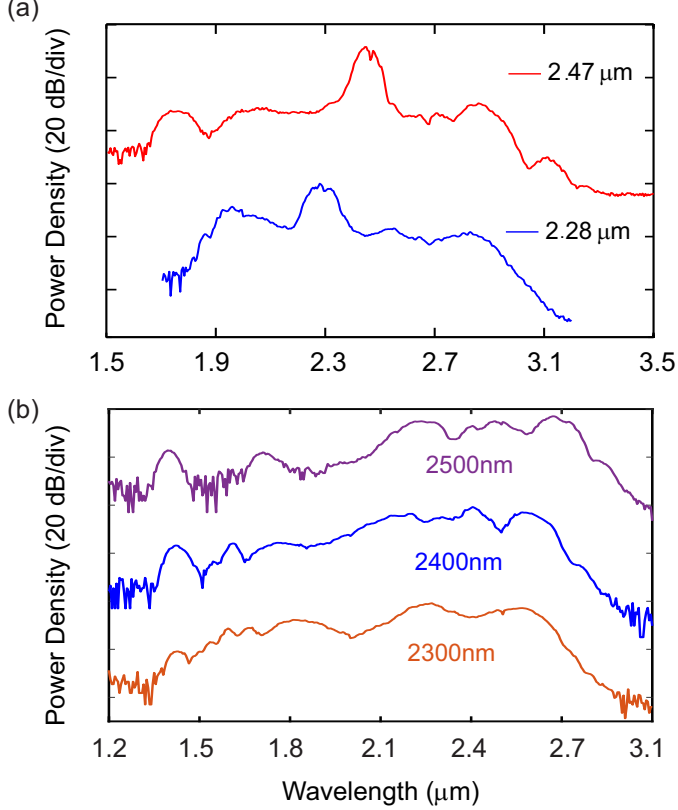

Fig. 2. (a) Supercontinuum spectra generated in a HPCVD hydrogenated amorphous silicon core fiber. (b) Supercontinuum spectra generated in a tapered MCD polysilicon core fiber.

\section{B. ZnSe Fibers}

One of the drawbacks of silicon for nonlinear optics is that the processes are limited to those based on the third order $\chi^{(3)}$ susceptibility. Thus the possibility to access the larger $\chi^{(2)}$ susceptibility in crystalline compound semiconductor core fibers is highly advantageous, not only to reduce the power thresholds, but also as it allows for the observation of processes such as second harmonic generation (SHG). In Fig. 3(a), we present the first demonstration of a visible (red) signal produced via SHG in a $\mathrm{ZnSe}$ core fiber, when it is pumped with a telecom source [8]. In order to satisfy phase matching the fiber was pumped in a WGM geometry, and the corresponding transmission spectrum is shown in the inset with the pump resonance highlighted in red. The position of the generated second harmonic light is in excellent agreement with the resonant pump at $1548 \mathrm{~nm}$ (i.e., $\lambda_{s}=1548 / 2 \sim 773.9 \mathrm{~nm}$ ) and the collected second harmonic power follows the expected quadratic dependence as the pump power is increased, as illustrated via the fitted curve in Fig. 3(b).
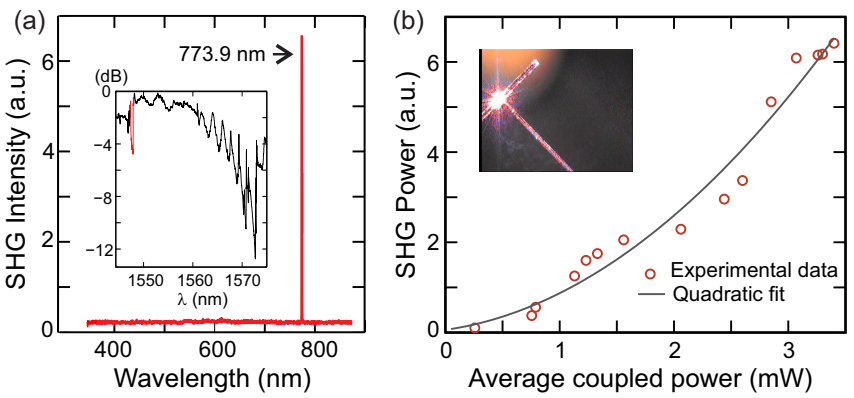

Fig. 3. (a) Measured second harmonic emission spectrum; inset displays the corresponding transmission spectrum showing the pump resonance at $\lambda \sim$ $1548 \mathrm{~nm}$ (red fit). (b) Plot of the measured second harmonic power as a function of the fundamental power dropped into the resonator. Inset shows a photograph of the generated visible second harmonic light.

\section{CONCLUSION}

Semiconductor fibers with various core materials and waveguide designs have been fabricated via chemical deposition and fiber drawing methods. Both these methods are now sufficiently advanced that the semiconductor fibers are regularly produced with optical transmission losses as low as a few $\mathrm{dB} / \mathrm{cm}$, which is enabling nonlinear characterizations and device demonstrators. Owing to the broad transparency windows of the semiconductor materials, these fibers could find use in applications extending beyond telecommunications and into important areas such as environmental sensing and quantum information

\section{ACKNOWLEDGMENT}

This work was supported by EPSRC.

\section{REFERENCES}

[1] J. Ballato, T. Hawkins, P. Foy, B. Yazgan-Kokuoz, C. McMillen, L. Burka, S. Morris, R. Stolen, and R. Rice, "Advancements in semiconductor core optical fiber," Opt. Fiber Technol. 16, 399-408 (2010).

[2] A. C. Peacock, J. R. Sparks, and N. Healy, "Semiconductor optical fibres: progress and opportunities," Laser Photonics Rev. 8, 53-72 (2014).

[3] F. H. Suhailin, L. Shen, N. Healy, L. Xiao, M. Jones, T. Hawkins, J. Ballato, U. J. Gibson, and A. C. Peacock, "Tapered polysilicon core fibers for nonlinear photonics," Opt. Lett. 41, 1360-1363 (2016).

[4] N. Healy, M. Fokine, Y. Franz, T. Hawkins, M. Jones, J. Ballato, A. C. Peacock, and U. J. Gibson, " $\mathrm{CO}_{2}$ laser-induced directional recrystallization to produce single crystal silicon-core optical fibers with low loss," Adv. Optical Mat. 4, 1004-1008 (2016).

[5] L. Shen, N. Healy, L. Xu, H. Y. Cheng, J. H. V. Price, J. V. Badding, and A. C. Peacock, "Four-wave mixing and octave-spanning supercontinuum generation in a small core hydrogenated amorphous silicon fiber pumped in the mid-infrared," Opt. Lett. 39, 5721-5724 (2014).

[6] H. Ren, L. Shen, J. Campling, A. F. J. Runge, O. Aktas, T. Hawkins, P. Horak, J. Ballato, U. Gibson, and A. C. Peacock, "Octave-spanning supercontinuum generation in a dispersion managed tapered crystalline silicon core fiber ," CLEO: Science and Innovations, Paper SM3D.5 (2018).

[7] Y. Franz, A. F. J. Runge, H. Ren, N. Healy, K. Ignatyev, M. Jones, T. Hawkins, J. Ballato, U. J. Gibson, A. C. Peacock, "Material properties of tapered crystalline silicon core fibers," Opt. Mater. Express 7, 20552061 (2017).

[8] N. Vukovic, N. Healy, J. R. Sparks, J. V. Badding, P. Horak, and A. C. Peacock, "Tunable continuous wave emission via phase-matched second harmonic generation in a ZnSe microcylindrical resonator,' Sci. Rep. 5, 11798 (2015). 